NUMERICAI ANALYSIS AND MATHEMATICAL MODELLING BANACH CENTER PUBLICATIONS, VOLUME 24 PWN-POLISH SCIENTIFIC PLIBLISHERS

WARSAW 1990

\title{
ON THE RELATIONSHIP BETWEEN DIFFERENCE AND PROJECTION-DIFFERENCE METHODS
}

\author{
N. A. STRELKOV
}

Yaroslavl Stale University, Yaroslavl, USSR

\section{Introduction}

The main purpose of this paper is to present a general point of view on the construction of projection-difference operators with prescribed properties. Another purpose is to construct projection-difference analogs of certain differential operators coinciding with their simplest difference analogs.

Projection-difference (finite element) methods combine the best properties of general projection (such as Ritz, Galerkin, moments, etc.) and difference methods. These methods lead to sparse algebraic problems and keep rate-of convergence optimality typical for projection methods.

In many cases the construction of these methods may be described in the following way. Let $a(\cdot, \cdot)$ be a coercive bilinear form on the linear normed space $V$ and let $V^{*}$ be adjoint space of $V$. For given $f \in V^{*}$ it is required to find $u \in V$ such that

$$
a(u, v)=f(v) \quad \text { for all } v \in V .
$$

Problems of the type (0.1) arise, for example, in finding generalized solutions of boundary value problems including nonlinear ones (certainly, in this case the form $a(\cdot, \cdot)$ is linear relative to the second argument only and satisfies some natural conditions).

Let now $V^{h}$ be a subspace of $V$ (finite-dimensional as a rule) and let $u^{h} \in V^{h}$ be a solution of problem

$$
a\left(u^{h}, v^{h}\right)=f\left(v^{h}\right) \quad \text { for all } v^{h} \in V^{h} .
$$

If $\left\{\psi_{a}^{h}\right\}$ is the basis of $V^{h}$ then the approximate solution is of the form

$$
u^{\prime \prime}=\sum_{\alpha} u_{a}^{h} \psi_{\alpha}^{h}
$$

Let us choose subspaces $V^{h} \subset V$ in such a way that two rather contradictory conditions are satisfied: 
(1) the sequence of subspaces $V^{\prime \prime}$ approximates $V$ in some sense,

(2) the resulting algebraic problem for determining unknown parameters $u_{x}^{h}$ has merits of difference method.

The method (0.2) for the solution of problem $(0.1)$ where subspaces $V^{\prime \prime}$ satisfy conditions (1)-(2) will be called PDM-projection-difference method (as a matter of fact it is the conformal finite element method [1]).

If we disengage ourselves from the fact that PDM is a particular case of general projection methods and treat PDMs as difference schemes whose coefficients are functionals of data then PDMs may be embedded into the class of general difference schemes considered in [2].

On the other hand, for the investigation of PDMs it is reasonable to disengage oneself from the grid character of these methods and treat PDMs as particular cases of general projection methods. Hence we may analyse PDM in the manner typical for general projection methods which is not connected with the concept of local approximation typical for difference schemes. By means of this approach it is possible to obtain error estimates in sufficiently strong norms under comparatively weak a priori assumptions about the smoothness of the unknown solution.

However, we must note that though difference and projection-difference methods are similar, nevertheless the projection-difference analogs of differential operators are comparatively extensively dispersed on the grid. For example, two-dimensional PDM for the equation $A^{2} u=f$ with Steklov's double averaging of characteristic functions of grid cells leads to a linear algebraic system with a linear combination of 25 unknowns in every equation whereas the pattern of usual difference analog of $\Delta^{2}$ consists of 13 points.

Thus PDMs possess an undisputable advantage over difference methods connected with the fact that all the treatments are carried out in the initial functional space and the questions of construction and investigation of resulting difference problems are essentially facilitated. At the same time PDMs are inferior to usual difference schemes in the simplicity of their structure.

In this connection the possibility of construction of PDMs whose structure coincides with that of usual difference schemes looks highly attractive. The existence of such PDMs allows us to establish new optimal error bounds for some well-known difference schemes. On the other hand, it is posible to use efficient iterative methods for the solution of resulting algebraic systems typical for difference approximations. Moreover, if the original problem is symmetric, positively defined, etc. then the corresponding property is automatically retained for the approximate problem. Let us also note that PDM application to problems with natural [3] boundary conditions leads to exactly those discrete approximations (frequently not so obvious) which guarantee the optimum rate of convergence. 
The first analyses of this kind were given in [4], where the projection-difference analog of 2-dimensional Laplace operator coinciding with its 5-point difference analog of "cross" type was constructed. The $n$-dimensional generalization of Courant's construction based on the special simplicial partitioning of $n$-dimensional parallelepiped is given in [5], [6] (in this connection see also [7]-[9]). These results are further developed in [8], [10]-[12] where PDMs coinciding with simplest difference schemes are constructed for certain 2-dimensional differential equations.

The general point of view on these PDMs is formulated in a theorem (see [13], [14]) which states necessary and sufficient conditions of coincidence of projection-difference analog of the given differential operator with its a priori given difference analog. Moreover, it is possible to use this theorem for the construction of PDMs coinciding with ordinary difference schemes.

The paper consists of 6 sections the first of which describes necessary and sufficient conditions of coincidence of difference and projection-difference operators. These conditions are similar to those of [13], [14] but they are more convenient for application and remain valid under weaker assumptions. The results of Section 1 are extensively used in other sections.

The family of prolongation operators of grid functions generating the projection-difference analog of $n$-dimensional Laplace operator coinciding with its simplest $(2 n+1)$-point difference analog of "cross" type is constructed in $\S 2$.

In Section 3, prolongation operators for a plane case are constructed; these operators ensure the coincidence of projection-difference and difference analogs of 4-th order differential operators (of biharmonic operator in particular).

The operator $B=D_{1}^{2 m}+D_{2}^{2 m}$ which is canonical for the construction of efficient two-stage iterative methods for the solution of $2 m$-th order boundary value problems is considered in Section 4; a special prolongation of grid functions is described so that projection-difference analog of operator $B$ coincides with its simplest $(4 m+1)$-point difference analog.

Some special interpolation formulae for entire functions of exponential type are constructed in $\S 5$. These formulae are a convenient tool for obtaining representations of entire functions which have zeroes of given multiplicity at the points $2 \pi \alpha, \alpha \in Z_{n}$ (just the same functions are Fourier images of functions generating prolongation operators with optimal approximation properties).

Finally, a complete description of projection-difference analogs of 2-dimensional Laplace operator which are generated by prolongation operators with optimal approximation properties is given in $\S 6$ by using results of $\S 1$ and $\S 5$.

\section{$\S 1$. The necessary and sufficient conditions of coincidence of difference and projection-difference operators}

Let us consider the following problem. Let $P(D)$ be a differential operator and let $Q(\partial)$ be a difference analog of $P(D)$. It is required to find the prolongation 
operator $R$ so that the projection-difference analog of $P(D)$ constructed by means of this prolongation operator coincides exactly with the operator $Q(\partial)$. In this section, conditions which are necessary and sufficient for such a coincidence are formulated in terms of Fourier transform of function generating the prolongation operator $R$.

Let $E_{n}$ be $n$-dimensional Euclidean space, $Z_{n}$ a set of integer vectors in $E_{n}$, $Z_{n}^{+}$a set of vectors in $Z_{n}$ with nonnegative components, $Z_{n}^{h}$ a rectangular grid in $E_{n}$ characterized by the vector $h=\left(h_{1}, \ldots, h_{n}\right) \in E_{n}$ with positive components; then any vector $\alpha=\left(\alpha_{1}, \ldots, \alpha_{n}\right) \in Z_{n}$ generates the node $\alpha^{h} \in Z_{n}^{h}$ of the form $\alpha^{\prime \prime}=\left(\alpha_{1} h_{1}, \ldots, \alpha_{n} h_{n}\right)$. If the grid function $u^{h}$ is defined in $Z_{n}^{h}$ then $u_{a}^{h}$ is the value of $u^{h}$ at the node $\alpha^{h} \in Z_{n}^{h}$.

Let $\varphi_{\alpha}^{h}(x)=\varphi(x / h-\alpha)$ where $\varphi$ is the characteristic function of the cube $\left\{x \in E_{n}:\left|x_{k}\right|<1 / 2, k=1, \ldots, n\right\}$.

Remark 1.1. Here and below for $x \in E_{n}, h \in E_{n}$ the expression $x / h$ denotes vector $\left(x_{1} / h_{1}, \ldots, x_{n} / h_{n}\right) \in E_{n}$.

For any grid function $u^{h}$ we define its piecewise constant prolongation $\bar{u}(x)$ by means of equality

$$
\bar{u}(x)=\sum_{\alpha \in Z_{n}} u_{\alpha}^{h} \varphi_{\alpha}^{h}(x)
$$

We shall identify grid functions and their piecewise constant prolongations of the type (1.1) below. Furthermore we shall assume that $\bar{u} \in L_{2}\left(E_{n}\right)$, in other words,

$$
\sum_{a \in Z_{n}}\left|u_{\alpha}^{h}\right|^{2}<\infty
$$

Alongside with piecewise constant prolongations (1.1) we shall consider smoother prolongations constructed as follows. Let $m$ be a natural number and let $\psi(x)$ be a function satisfying the following conditions:

(A) its support is compact;

(B) $\quad \psi \in W_{2}^{m}\left(E_{n}\right)$.

Here $W_{2}^{\prime \prime \prime}\left(E_{n}\right)$ is the Sobolev space (see, for instance, [16]). The function $\psi$ generates the linear prolongation operator $R$ such that

$$
R \bar{u}(x)=\sum_{\alpha \in Z_{n}} u_{\alpha}^{h} \psi_{\alpha}^{\prime \prime}(x)
$$

for every grid function $\bar{u}$ of the form (1.1) where

$$
\psi_{\alpha}^{h}(x)=\psi\left(\frac{x}{h}-\alpha\right) .
$$


Let $P(D)$ be a linear differential operator with constant coefficients of the following form:

$$
P(D)=\sum_{|\gamma| \leqslant 2 m} a_{\gamma} D^{\gamma}
$$

The order of $P$ is less or equal to $2 m$ (recall that $\psi \in W_{2}^{m}\left(E_{n}\right)$ ).

Remark 1.2. In (1.4) and below $\gamma=\left(\gamma_{1}, \ldots, \gamma_{n}\right) \in Z_{n}^{+},|\gamma|=\gamma_{1}+\ldots+\gamma_{n}$, $D^{\gamma}=D_{1}^{\gamma_{1}} \ldots D_{n}^{\gamma_{n}}, D_{k}=\frac{\partial}{\partial x_{k}}, k=1, \ldots, n$.

Let us consider difference operator $Q(\hat{\partial})$ with constant but possibly depending on $h$ coefficients:

$$
Q(\hat{\partial})=\sum_{\gamma} b_{\gamma} \partial^{\gamma}
$$

where $\hat{\partial}^{\gamma}=\hat{\partial}_{1}^{\gamma_{1}} \ldots \hat{\partial}_{n}^{\gamma_{n}}, \hat{\partial}_{k} f(x)=h_{k}^{-1}\left[f\left(x+\frac{1}{2} h_{k} e_{k}\right)-f\left(x-\frac{1}{2} h_{k} e_{k}\right)\right],\left\{e_{1}, \ldots, e_{n}\right\}$ is orthonormal basis for $E_{n}$.

Let us consider the problem of finding function $\psi$ with properties $\mathrm{A}$ and $B$ such that prolongations of the form (1.2) generated by this function satisfy also the following conditions of approximation and isometry:

(C) for any $u \in W_{2}^{m+1}\left(E_{n}\right)$ there exists a grid function $\bar{u}$ of the form (1.1) such that

$$
\begin{aligned}
\|\bar{u}\|_{L_{2}\left(E_{n}\right)} & \leqslant K\|u\|_{L_{2}\left(E_{n}\right)}, \\
\|u-R \bar{u}\|_{W_{2}^{s}\left(E_{n}\right)} & \leqslant C_{s}|h|^{m+1-s}\|u\|_{W_{2}^{m+1}\left(E_{n}\right)}, \quad 0 \leqslant s \leqslant m .
\end{aligned}
$$

where $K$ and $C_{s}$ do not depend on $u$ and $h$;

(D) for all grid functions $\bar{u}$ and $\bar{v}$

$$
(P(D) R \bar{u}, R \bar{v})_{L_{2}\left(E_{n}\right)}=(Q(\hat{\partial}) \bar{u}, \bar{v})_{L_{2}\left(E_{n}\right)} .
$$

Remark 1.3. In (1.6) and below there is the expression $(P(D) R \bar{u}, R \bar{v})_{L_{2}\left(E_{n}\right)}$; let us explain its sense. The matter is that $R \bar{u} \in W_{2}^{m}\left(E_{n}\right)$ and the order of operator $P(D)$ is equal to $2 m$ therefore generally speaking $P(D) R \bar{u} \notin L_{2}\left(E_{n}\right)$. Thus the expression $(P(D) R \bar{u}, R \bar{v})_{L_{2}\left(E_{n}\right)}$ must be treated as the value of $P(D) R \bar{u} \in W_{2}^{-m}\left(E_{n}\right)=\left(W_{2}^{n}\left(E_{n}\right)\right)^{*}$ at the element $R \bar{v} \in W_{2}^{m}\left(E_{n}\right)$. In other words it is necessary to apply Green's formula and to write $(P(D) R \bar{u}, R \bar{v})_{L_{2}\left(E_{n}\right)}$ in the form

$$
(P(D) R \bar{u}, R \bar{v})_{L_{2}\left(E_{n}\right)}=\sum_{j}\left(A_{j}(D) R \bar{u}, B_{j}^{*}(D) R \bar{v}\right)_{L_{2}\left(E_{n}\right)}
$$

where $\sum_{j} A_{j}(D) B_{j}(D)=P(D)$ and orders of differential operators $A_{j}(D)$ and $B_{j}(D)$ are not greater than $m$. Then $A_{j}(D) R \bar{u} \in L_{2}\left(E_{n}\right), B_{j}(D) R \bar{v} \in L_{2}\left(E_{n}\right)$ and the right-hand side of (1.7) is the sum of ordinary scalar products in $L_{2}\left(E_{n}\right)$. 
Remark 1.4. The identity (1.6) means the coincidence of the projection-difference analog of operator $P(D)$ constructed with the help of prolongation operator $R$ with the difference operator $Q(\partial)$. Really if in $(0.1)$ $V=W_{2}^{n}\left(E_{n}\right), a(u, v)=(P(D) u, v)_{L_{2}}$ (see remark 1.3) and $V^{h}=\left\{\psi_{\alpha}^{h}\right\}_{\alpha \in Z_{n}}$ where $\psi_{\alpha}^{h}$ is of the form (1.3) then (0.2) and (1.6) imply that if $u^{h}=R \bar{u} \in V^{h}$ is the approximate solution then for the grid function $\bar{u}$ the identity $(Q(\hat{\partial}) \bar{u}, \bar{v})_{L_{2}}=f(R \bar{v})$ is true for all grid functions $\bar{v}$ of the type (1.1). The latter identity means that the unknown grid function $\vec{u}$ is the solution of difference problem with operator $Q(\hat{\partial})$.

There are a number of prolongations satisfying conditions $\mathrm{A}, \mathrm{B}, \mathrm{C}$ (see for instance [1], [15]); at the same time the prolongation operators satisfying also the condition $D$ which is important for the numerical realisation of PDM and means the coincidence of projection-difference analog of $P(D)$ with premeditated difference operator $Q(\delta)$ are constructed for some particular cases only (these cases were mentioned above).

The next assertion in terms of Fourier transform of function $\psi$ formulates conditions which are necessary and sufficient for the implementation of requirements $\mathrm{A}, \mathrm{B}, \mathrm{C}, \mathrm{D}$.

Let

$$
\hat{\psi}(x)=\int_{E_{n}} \psi(t) e^{-(t, x)} d t
$$

be the Fourier transform of $\psi$ (here $\left.(t, x)=t_{1} x_{1}+\ldots+t_{n} x_{n}\right)$.

THEOREM 1.1. The conditions $\mathrm{A}, \mathrm{B}, \mathrm{C}, \mathrm{D}$ are equivalent to the following four conditions respectively:

$\left(\mathrm{A}^{\prime}\right) \hat{\psi}(z)$ is an entire function of exponential type [16];

(B) $\hat{\psi}(x)\left(1+|x|^{2}\right)^{m / 2} \in L_{2}\left(E_{n}\right)$;

(C) $\hat{\psi}(0) \neq 0$ and $\hat{\psi}$ has zeroes of multiplicity at least $m+1$ at the points $2 \pi \alpha, 0 \neq \alpha \in Z_{n}$, i.e.

$D^{\beta} \hat{\psi}(2 \pi \alpha)=0$ for any $\beta \in Z_{n}^{+},|\beta| \leqslant m$ and for all $\alpha \in Z_{n}, \alpha \neq 0$;

(D) $\sum_{\alpha \in Z_{n}} F(x+2 \pi \alpha)=G(x)$ for all $x \in E_{n}$ where

$$
\begin{gathered}
F(x)=P\left(\frac{i x}{h}\right)|\hat{\psi}(x)|^{2} \\
G(x)=\sum_{\gamma} b_{\gamma}\left(\frac{i \sin x}{h}\right)^{\gamma}\left(\cos \frac{x}{2}\right)^{-2[\gamma / 2]}
\end{gathered}
$$

Remark 1.5. In (1.8), (1.9) and below for $x \in E_{n}, y \in E_{n}$ the following notations are used:

$$
\begin{gathered}
x^{y}=x_{1}^{y_{1}} \ldots x_{n}^{y_{n}} \in E_{1}, \quad \sin x=\left(\sin x_{1}, \ldots, \sin x_{n}\right) \in E_{n}, \\
\cos x=\left(\cos x_{1}, \ldots, \cos x_{n}\right) \in E_{n}, \quad[x]=\left(\left[x_{1}\right], \ldots,\left[x_{n}\right]\right) \in E_{n}
\end{gathered}
$$


where $\left[x_{k}\right]$ is the integral part of $x_{k}$,

$$
\begin{gathered}
(x, y)=x_{1} y_{1}+\ldots+x_{n} y_{n} \in E_{1}, \quad x y=\left(x_{1} y_{1}, \ldots, x_{n} y_{n}\right) \in E_{n}, \\
\frac{x}{y}=\left(\frac{x_{1}}{y_{1}}, \ldots, \frac{x_{n}}{y_{n}}\right) \in E_{n} .
\end{gathered}
$$

Remark 1.6. Note that if $Q$ is a symmetric operator, i.e.

$$
Q(\partial)=\sum_{\gamma} c_{\gamma} \delta^{2 \gamma}
$$

then

$$
G(x)=\sum_{\gamma} c_{\gamma}\left(\frac{2 i \sin \frac{x}{2}}{h}\right)^{2 \gamma}=Q\left(\frac{2 i \sin \frac{x}{2}}{h}\right) .
$$

Proof of Theorem 1.1. First of all note that the equivalence of conditions $A$ and $A^{\prime}$ follows from the Paley-Wiener theorem (see [16], p. 130); conditions $B$ and $B^{\prime}$ are none other than two equivalent definitions of Sobolev's space $W_{2}^{m}\left(E_{n}\right)$ (see for instance [17]). As for the equivalence of $\mathrm{C}$ and $\mathrm{C}^{\prime}$ then it is the Strange-Fix theorem (see [15], [18], [19]).

Thus it remains to prove the equivalence of conditions $D$ and $D^{\prime}$. Using the Plancherel theorem (see for instance [16]) we deduce that condition $D$ is equivalent to the fact that identity

$$
(\mathscr{F} P(D) R \bar{u}, \mathscr{F} R \bar{v})_{L_{n}\left(E_{n}\right)}=(\mathscr{F} Q(\hat{\partial}) \bar{u}, \mathscr{F} \bar{v})_{L_{2}\left(E_{n}\right)}
$$

is true for all grid functions $\bar{u}$ and $\tilde{v}$ of the form (1.1) where $\mathscr{F}$ is the Fourier transform operator. Since

$$
\begin{gathered}
\mathscr{F}\left[D_{k} g\right](t)=\int_{E_{n}} D_{k} g(x) e^{-i(t, x)} d x=i t_{k} \dot{\mathscr{F}} g(t), \\
\mathscr{F} \psi_{\alpha}^{h}(t)=\int_{E_{n}} \dot{\psi}\left(\frac{x}{h}-\alpha\right) e^{-i(t, x)} d x \\
=\bar{h} \int_{E_{n}} \psi(y) e^{-i(t, y h+\alpha h)} d y=\bar{h} e^{-i(t, \alpha h)} \mathscr{F} \psi(t h), \\
\mathscr{F} \varphi_{\alpha}^{\prime \prime}(x)=\bar{h} e^{-i(r, \alpha h)} \mathscr{F} \varphi(t h),
\end{gathered}
$$

where $\bar{h}=h_{1} \ldots h_{n}$ and

$$
\begin{aligned}
\mathscr{F}\left[\hat{\partial}_{k} g\right](t) & =\int_{E_{n}} h_{k}^{-1}\left[g\left(x+\frac{1}{2} h_{k} e_{k}\right)-g\left(x-\frac{1}{2} h_{k} e_{k}\right)\right] e^{-i(t, x)} d x \\
& =h_{k}^{-1} \int_{E_{n}} g(y)\left[e^{-i\left(t, y-\frac{1}{2} h_{k} e_{k}\right)}-e^{-i\left(t, y+\frac{1}{2} h_{k} e_{k}\right)}\right] d y \\
& =2 i h_{k}^{-1} \sin \frac{t_{k} h_{k}}{2} \mathscr{F} g(t),
\end{aligned}
$$


the condition (1.10) is equivalent to the fact that the identity

$$
\begin{aligned}
\sum_{\alpha \in Z_{n}} \sum_{\beta \in Z_{n}} u_{\alpha} \bar{v}_{\beta} \int_{E_{n}} P(i t) e^{-i(t,(\alpha-\beta) h)}|\mathscr{F} \psi(t h)|^{2} d t \\
=\sum_{\alpha \in Z_{n}} \sum_{\beta \in Z_{n}} u_{\alpha} \bar{v}_{\beta} \int_{E_{n}} Q\left(\frac{2 i \sin (t h) / 2}{h}\right) e^{-i(t,(\alpha-\beta) h)}|\mathcal{F} \varphi(t h)|^{2} d t
\end{aligned}
$$

is true for all $u_{\alpha}, v_{\beta}$. The last condition is equivalent to the identity

$$
\int_{E_{n}}\left\{P(i t)|\mathscr{F} \psi(t h)|^{2}-Q\left(\frac{2 i \sin (t h) / 2}{h}\right)|\mathscr{F} \varphi(t h)|^{2}\right\} e^{-i(t, \nu h)} d t=0
$$

for any $\gamma \in Z_{n}$ or (which is the same)

$$
\int_{\Omega}\left\{\sum_{\alpha \in Z_{n}} H(x+2 \pi \alpha)\right\} e^{-i(x, y)} d x=0
$$

for all $\gamma \in Z_{n}$ where

$$
\begin{gathered}
H(x)=P\left(\frac{i x}{h}\right)|\mathscr{F} \psi(x)|^{2}-Q\left(\frac{2 i \sin x / 2}{h}\right)|\mathscr{F} \varphi(x)|^{2}, \\
\Omega=\left\{x \in E_{n}:-\pi<x_{k}<\pi, k=1, \ldots, n\right\} .
\end{gathered}
$$

Since $H \in L_{1}\left(E_{n}\right)$, it follows that $\sum_{\alpha \in Z_{n}} H(x+2 \pi \alpha) \in L_{1}(\Omega)$ and from the completeness of the system $\left\{e^{-i(x, \gamma)}\right\}_{\gamma \in Z_{n}}$ in $L_{1}(\Omega)$ and (1.11) we deduce that (1.10) is equivalent to the identity

$$
\sum_{\alpha \in Z_{n}} H(x+2 \pi \alpha)=0, \quad x \in \Omega
$$

It is easy to calculate that

$$
\mathscr{F} \varphi(x)=\left(\frac{2 \sin x / 2}{x}\right)^{\hat{1}}
$$

(here and below for any $\left.a \in E_{1} \vec{a}=(a, \ldots, a) \in E_{n}\right)$. Thus, using (1.5), we find that

$$
\begin{aligned}
\sum_{\alpha \in Z_{n}} Q\left(\frac{2 i \sin (x+2 \pi \alpha) / 2}{h}\right) \mid & \left.\mathscr{F} \varphi(x+2 \pi \alpha)\right|^{2} \\
& =\sum_{\gamma} b_{\gamma} \sum_{\alpha \in Z_{n}}\left[\frac{2 i \sin (x / 2+\pi \alpha)}{h}\right]^{\gamma}\left[\frac{\sin (x / 2+\pi \alpha)}{\frac{x}{2}+\pi \alpha}\right]^{\overrightarrow{2}} \\
& =\sum_{\gamma} b_{\gamma}\left(\frac{2 i \sin x / 2}{h}\right)^{\gamma} \sum_{\alpha \in Z_{n}}(-1)^{(\alpha, \gamma)} \frac{\left(\frac{\sin x / 2}{x}\right)^{\overrightarrow{2}}}{\frac{x}{2}+\pi \alpha}
\end{aligned}
$$


Since

$$
\sum_{k=-\infty}^{\infty} \frac{\sin ^{2} \xi}{(\xi+\pi k)^{2}}=1, \quad \sum_{k=-\infty}^{\infty} \frac{(-1)^{k} \sin ^{2} \xi}{(\xi+\pi k)^{2}}=\cos \xi
$$

(see, for instance, [20]), we have

$$
\sum_{a \in Z_{n}} Q\left(\frac{2 i \sin (x+2 \pi \alpha) / 2}{h}\right)|\mathscr{F} \varphi(x+2 \pi \alpha)|^{2}=\sum_{\gamma} b_{\gamma}\left(\frac{i \sin x}{h}\right)^{\gamma}\left(\cos \frac{x}{2}\right)^{-2[\gamma / 2]}
$$

The rest of the proof follows from (1.12).

Remark 1.7. If we require that the support of $\psi$ in the condition $A$ belongs to the cube $\left\{x \in E_{n}:\left|x_{k}\right| \leqslant(m+1) / 2, k=1, \ldots, n\right\}$ (this is equivalent to the following modification of the condition $A^{\prime}: \hat{\psi}$ is the entire function of exponential type $(m+1) / 2)$ then $\mathrm{D}^{\prime}$ is equivalent to the equalities

$$
\sum_{\alpha \in Z_{n}} D^{\beta} F(2 \pi \alpha)=D^{\beta} G(0)
$$

for any $\beta \in Z_{n}^{+}$such that $0 \leqslant \beta_{k} \leqslant 2 m, k=1, \ldots, n$ (see [14]). Note that owing to the condition $C^{\prime}$ some of derivatives in the left part of (1.14) are equal to zero for $\alpha \neq 0$.

Remark 1.8. If $Q(\delta)$ is the operator of the type (1.5) and

$$
Q(\partial, \bar{\partial}, \hat{\partial})=\sum_{\gamma} b_{\gamma}(\bar{\partial} \partial)^{[\gamma / 2]} \tilde{\partial}^{\tilde{\gamma}-2[\gamma / 2]}
$$

where $b_{y}$ are coefficients of $Q(\hat{\partial})$ and difference operators $\partial_{k}, \bar{\partial}_{k}, \tilde{\partial}_{k}$ are defined by the equalities

$$
\partial_{k} u_{\alpha}^{h}=h_{k}^{-1}\left(u_{\alpha+e_{k}}^{h}-u_{\alpha}^{h}\right), \quad \bar{\partial}_{k} u_{\alpha}^{h}=h_{k}^{-1}\left(u_{\alpha}^{h}-u_{\alpha-e_{k}}^{h}\right), \quad \tilde{\partial}_{k}=\frac{1}{2}\left(\partial_{k}+\bar{\partial}_{k}\right)
$$

then for any $\alpha \in Z_{n}$ and for all grid functions $u^{h}$,

$$
Q(\partial, \bar{\partial}, \tilde{\partial}) u_{\alpha}^{h}=\bar{h}^{-1}\left(Q(\partial) \bar{u}, \varphi_{\alpha}^{h}\right)_{L_{2}\left(E_{n}\right)} .
$$

The latter equality establishes a connection between "traditional" difference operators $Q(\partial, \bar{\partial}, \widetilde{\partial})$ and operators $Q(\hat{\partial})$ acting on piecewise linear functions of the form (1.1). The utilization of (1.15) allows us to reformulate Theorem 1.1 in terms of operator $Q(\partial, \bar{\partial}, \tilde{\partial})$ (only the formulation of the condition $\mathrm{D}$ will be new).

\section{§2. Piecewise linear prolongations generating the simplest difference analog of Laplace operator}

The $n$-dimensional generalization of Courant's construction [4] will be considered in this section. More precisely, we shall construct the family of 
prolongation operators generating projection-difference analogs of $n$-dimensional Laplace operator coinciding with its simplest difference analog of "cross" type.

Let us consider the function

$$
g(t)=\frac{2 \sin t / 2}{t}
$$

Note that $g(0)=1, g(2 \pi k)=0,0 \neq k \in Z_{1}, g^{\prime}(0)=0$. Let us define the functions

$$
\psi(z)=g\left(z_{1}\right) \ldots g\left(z_{n}\right) g\left(\gamma_{1} z_{1}+\ldots+\gamma_{n} z_{n}\right)
$$

depending on parameter $\gamma=\left(\gamma_{1}, \ldots, \gamma_{n}\right) \in Z_{n}$ where each of $\gamma_{k}$ is equal to one of two values: 1 or -1 .

It is easy to see that $\psi(z)$ is the entire function of exponential type 1 [16] and $\hat{\psi}(x)\left(1+|x|^{2}\right)^{1 / 2} \in L_{2}\left(E_{n}\right), \psi(0)=1, \hat{\psi}(2 \pi \alpha)=0$ for any $\alpha \in Z_{n}, \alpha \neq 0$ while

$$
\begin{aligned}
D_{k} \psi(2 \pi \alpha)= & g\left(2 \pi \alpha_{1}\right) \ldots g\left(2 \pi \alpha_{n}\right)\left[\gamma_{k} g^{\prime}\left(2 \pi\left(\gamma_{1} \alpha_{1}+\ldots+\gamma_{n} \alpha_{n}\right)\right)\right. \\
& \left.+\frac{g^{\prime}\left(2 \pi \alpha_{k}\right)}{g\left(2 \pi \alpha_{k}\right)} g\left(2 \pi\left(\gamma_{1} \alpha_{1}+\ldots+\gamma_{n} \alpha_{n}\right)\right)\right]=0
\end{aligned}
$$

for all $\alpha \in Z_{n}, k=1, \ldots, n$. Thus the function $\hat{\psi}$ satisfies conditions $\mathrm{A}^{\prime}, \mathrm{B}^{\prime}, \mathrm{C}^{\prime} \S 1$ with $m=1$.

Now let

$$
\begin{aligned}
& P(D)=\Delta=D_{1}^{2}+\ldots+D_{n}^{2}, \\
& Q(\hat{\partial})=\hat{\Delta}=\delta_{1}^{2}+\ldots+\hat{\partial}_{n}^{2} .
\end{aligned}
$$

Then the functions $F$ and $G$ of the types (1.8), (1.9) are

$$
\begin{gathered}
F(x)=-\left[\left(\frac{x_{1}}{h_{1}}\right)^{2}+\ldots+\left(\frac{x_{n}}{h_{n}}\right)^{2}\right] g^{2}\left(x_{1}\right) \ldots g^{2}\left(x_{n}\right) g^{2}\left(\gamma_{1} x_{1}+\ldots+\gamma_{n} x_{n}\right), \\
G(x)=-\left[\left(\frac{2}{h_{1}} \sin \frac{x_{1}}{2}\right)^{2}+\ldots+\left(\frac{2}{h_{n}} \sin \frac{x_{n}}{2}\right)^{2}\right] .
\end{gathered}
$$

Let us prove that

$$
\sum_{\alpha \in Z_{n}} F(x+2 \pi \alpha)=G(x)
$$

It is obvious that the last equality holds if

$$
\begin{aligned}
\sum_{\alpha \in Z_{n}}\left(\frac{x_{k}}{2}+\pi \alpha_{k}\right)^{2} g^{2}\left(\sum_{s=1}^{n} \gamma_{s} x_{s}+2 \pi \sum_{s=1}^{n} \gamma_{s} \alpha_{s}\right) \prod_{s=1}^{n} g^{2}\left(x_{s}+2 \pi \alpha_{s}\right) & \\
& =\sin ^{2} \frac{x_{k}}{2} \quad \text { for all } k=1, \ldots, n
\end{aligned}
$$


While

$$
\left(\frac{x_{k}}{2}+\pi \alpha_{k}\right)^{2} g^{2}\left(x_{k}+2 \pi \alpha_{k}\right)=\sin ^{2} \frac{x_{k}}{2}
$$

does not depend on $\alpha_{k}$ and

$$
\sum_{a_{k}=-\infty}^{\infty} g^{2}\left(\sum_{s=1}^{n} \gamma_{s} x_{s}+2 \pi \sum_{s=1}^{n} \gamma_{s} \alpha_{s}\right)=1
$$

(see (1.13)) then by calculating the sum in the left-hand side of (2.6) and taking into account that

$$
\sum_{\alpha_{s}=-\infty}^{\infty} g^{2}\left(x_{s}+2 \pi \alpha_{s}\right)=1
$$

we prove equality (2.6). Therefore the condition $D^{\prime}$ is satisfied for the functions $F$ and $G$ of the forms (2.4), (2.5).

Thus the conditions $\mathrm{A}^{\prime}-\mathrm{D}^{\prime}$ and also the conditions $\mathrm{A}-\mathrm{D}$ due to Theorem 1.1 are satisfied for $m=1$ and for operators $P$ and $Q$ of the forms (2.2), (2.3). Hence the prolongation operator satisfying conditions stated at the beginning of this section is constructed.

For more details on properties of this prolongation operator see [6] (the case $\gamma_{1}=\ldots=\gamma_{n}=1$ ) and [8], [9] (the case of arbitrary $\gamma$ ).

Remark 2.1. The function $\psi$ of the form (2.1) generates the prolongation operator $R$ acting on the grid functions $\bar{u}$ (see (1.1), (1.2)) such that

$$
R \bar{u}(x)=\int_{-1 / 2}^{1 / 2} \bar{u}(x+h \gamma t) d t .
$$

The vector $\gamma=\left(\gamma_{1}, \ldots, \gamma_{n}\right)$ with components \pm 1 defines the direction of interval of averaging piecewise constant function $\bar{u}$.

Remark 2.2. Since the function $g(t)$ is even and $\gamma_{s}= \pm 1$, there exist exactly $2^{n-1}$ vectors $\gamma$ generating different functions $\psi$ of the form (2.1) (we may, for example, fix $\gamma_{1}=1$; then there exist $2^{n-1}$ variants of distributing 1 or -1 among other $n-1$ components). Any vector $\gamma$ of this type generates somc simplicial partition of every grid cell into $n$ ! elementary simplexes and the function $R \bar{u}$ is linear in every elementary simplex. Moreover, the properties $A-D$ remain valid if the triangulations of all grid cells are generated by not the some fixed vector $\gamma$ but it is possible to vary vector $\gamma$ with the components \pm 1 from cell to cell (here is the analogy with 2-dimensional case where it is possible to draw one of two diagonals in every grid cell).

\section{§3. The projection-difference analog of biharmonic operator coinciding with its simplest difference analog.}

Let us consider 2-dimensional case in detail. We shall use the notations of previous sections for $n=2$. 
Let us define the following averaging operators:

$$
\begin{aligned}
r f(x) & =\int_{-1 / 2}^{1 / 2} \int_{-1 / 2}^{1 / 2} f\left(x_{1}+t_{1} h_{1}, x_{2}+t_{2} h_{2}\right) d t_{1} d t_{2} \\
S_{1} f(x) & =\int_{-1 / 2}^{1 / 2} f\left(x_{1}+t h_{1}, x_{2}+t h_{2}\right) d t \\
S_{2} f(x) & =\int_{-1 / 2}^{1 / 2} f\left(x_{1}+t h_{1}, x_{2}-t h_{2}\right) d t
\end{aligned}
$$

Then, according to the results of [8], [9], for the arbitrary function $\bar{u}$ of the form (1.1) $r \bar{u}$ is bilinear and $S_{1} \bar{u}, S_{2} \bar{u}$ are piecewise linear prolongations of the grid function $\bar{u}$. Note that $S_{1} \bar{u}$ and $S_{2} \bar{u}$ correspond to a pair of different natural triangulations of grid cells.

Let us consider averaging operator

$$
R=(1+c) r S_{1}+(1-c) r S_{2}-S_{1} S_{2}
$$

depending on the real parameter $c$. If $\bar{u}$ is a grid function of the form (1.1) then $R \bar{u}$ is a cubic piecewise-polynomial function (see [8], [9]). In addition, $R \bar{u}$ is of the form (1.2) and the Fourier image of the function $\psi$ generating the prolongation operator $R$ is

$$
\begin{aligned}
\hat{\psi}(z)= & g^{2}\left(z_{1}\right) g^{2}\left(z_{2}\right)\left((1+c) g\left(z_{1}+z_{2}\right)+(1-c) g\left(z_{1}-z_{2}\right)\right) \\
& -g\left(z_{1}\right) g\left(z_{2}\right) g\left(z_{1}+z_{2}\right) g\left(z_{1}-z_{2}\right),
\end{aligned}
$$

where $z=\left(z_{1}, z_{2}\right), g(t)=(2 \sin (t / 2)) / t$.

In view of (3.2) $\bar{\psi}$ is the entire function of exponential type $3 / 2$ and $\hat{\psi}(x)\left(1+|x|^{2}\right) \in L_{2}\left(E_{2}\right)$, i.e. for every $c$ the conditions $\mathrm{A}^{\prime}$ and $\mathbf{B}^{\prime} \S 1$ are satisfied for $m=2$. It is easy to verify that for every $c \psi$ satisfies the condition $C^{\prime}$ for $m=2$.

Let us now find the parameter $c$ to satisfy the condition $D^{\prime}$ for the case

$$
P(D)=\Lambda^{2}=\left(D_{1}^{2}+D_{2}^{2}\right)^{2}, \quad Q(\hat{\partial})=\hat{\Delta}^{2}=\left(\hat{\partial}_{1}^{2}+\hat{\partial}_{2}^{2}\right)^{2} .
$$

Let $h_{1}=h_{2}=h$. Then

$$
\begin{aligned}
& F(x)=h^{-4}\left(x_{1}^{2}+x_{2}^{2}\right)^{2}|\hat{\psi}(x)|^{2}, \\
& G(x)=h^{-4} \cdot 2^{4}\left(\sin ^{2} \frac{x_{1}}{2}+\sin ^{2} \frac{x_{2}}{2}\right)^{2}
\end{aligned}
$$

hence the condition $\mathrm{D}^{\prime}$ is equivalent to the equality

$$
\sum_{\alpha \in Z_{2}}\left[\left(\frac{x_{1}}{2}+\pi \alpha_{1}\right)^{2}+\left(\frac{x_{2}}{2}+\pi \alpha_{2}\right)^{2}\right]^{2}|\hat{\psi}(x+2 \pi \alpha)|^{2}=\left(\sin ^{2} \frac{x_{1}}{2}+\sin ^{2} \frac{x_{2}}{2}\right)^{2}
$$

By means of elementary but rather lengthy calculations one can show that the equality (3.3) is true if and only if $c^{2}=1 / 2$. 
Thus the prolongation operator $R$ of the form (3.1) with $c^{2}=1 / 2$ generates projection-difference analog of biharmonic operator coinciding with its simplest 13-point difference analog.

Remark 3.1. By means of analogous arguments we can obtain the following assertion. If

$$
R=r\left((1-2 a) r+(a+b) S_{1}+(a-b) S_{2}\right)
$$

where $a^{2}=11 / 4, b^{2}=3 / 4$ then the conditions $\mathrm{A}, \mathrm{B}, \mathrm{C}, \mathrm{D}$ are satisfied with $m=2, P(D)=D_{1}^{4}+D_{2}^{4}, Q(\delta)=\delta_{1}^{4}+\hat{\partial}_{2}^{4}$, i.e. the prolongation operator (3.4) generates projection-difference analog of the operator $D_{1}^{4}+D_{2}^{4}$ coinciding with its simplest 9-point difference analog (see [8], [14], for details).

Remark 3.2. By utilizing properties of the operator (3.1) it is possible to obtain new error estimates of the difference method for the biharmonic equation. These estimates are optimal for natural [3] boundary conditions and near optimal for the main boundary conditions (see [8], [11]).

\section{§4. On projection-difference analogs}

of the operator $D_{1}^{2 m}+D_{2}^{2 m}$

In this section the prolongation operator satisfying the conditions $A, B, C$, D $\S 1$ with the operators

$$
P(D)=D_{1}^{2 m}+D_{2}^{2 m}, \quad Q(\delta)=\hat{\delta}_{1}^{2 m}+\hat{\partial}_{2}^{2 m}
$$

is constructed for every $m$.

Let us define the function

$$
\psi(z)=\left[g\left(z_{1}\right) g\left(z_{2}\right)\right]^{m+1}\left(1+\sum_{s=1}^{m} b_{s} \prod_{k=2}^{s+1} \operatorname{tg} \frac{z_{1}}{2^{k}} \operatorname{tg} \frac{z_{2}}{2^{k}}\right)
$$

depending on an arbitrary set of real numbers $b_{1}, \ldots, b_{m}$, where $z=\left(z_{1}, z_{2}\right)$, $g(t)=(2 \sin (t / 2)) / t$.

Using some properties of the function $g$, we can easily verify that for arbitrary $b_{1}, \ldots, b_{m} \hat{\psi}(z)$ satisfies the conditions $\mathbf{A}^{\prime}, \mathbf{B}^{\prime}, \mathrm{C}^{\prime} \S 1$ (note that the type of $\psi$ is equal to $(m+1) / 2)$.

Let us now determine the parameters $b_{1}, \ldots, b_{m}$ such that for the operators $P(D)$ and $Q(\delta)$ of the form (4.1) the condition $\mathrm{D}^{\prime}$ is satisfied. This condition implies that

$$
\sum_{x \in Z_{n}}\left[\left(\frac{x_{1}}{2}+\pi \alpha_{1}\right)^{2 m}+\left(\frac{x_{2}}{2}+\pi \alpha_{2}\right)^{2 m}\right]|\hat{\psi}(x+2 \pi \alpha)|^{2}=\sin ^{2 m} \frac{x_{1}}{2}+\sin ^{2 m} \frac{x_{2}}{2} .
$$

For the calculation of the sum in the left-hand side of (4.3) we need the following assertion (which is also of an independent importance). 
LEMMA 4.1. Let $p$ be a nonnegative integer, $\alpha_{1}, \ldots, \alpha_{p}$ be a set of integers each of which is equal to 0,1 or 2 . Then

$$
\sum_{k=-\infty}^{\infty}\left(\frac{\sin z}{z+\pi k}\right)^{2} \prod_{s=1}^{p} \operatorname{tg}^{\alpha_{s}} \frac{z+\pi k}{2^{s}}= \begin{cases}1 & \text { if no one of } \alpha_{s} \text { is equal to } 1 \\ 0 & \text { otherwise. }\end{cases}
$$

Proof. If $p=0$ then the assertion is true, because both sides of (4.4) are equal to 1. Let us assume that (4.4) is true for every $p \leqslant M$ and prove it for $p=M+1$. Calculating the sum in the left-hand side of (4.4) separately for even and odd $k$, we have

$$
\begin{aligned}
\sum_{k=-\infty}^{\infty} & \left(\frac{\sin z}{z+\pi k}\right)^{2} \prod_{s=1}^{M+1} \operatorname{tg}^{\alpha_{s}} \frac{z+\pi k}{2^{s}} \\
= & \sum_{k=-\infty}^{\infty}\left(\frac{\sin z}{z+2 \pi k}\right)^{2} \prod_{s=0}^{M} \operatorname{tg}^{\alpha_{s+1}} \frac{(z / 2)+\pi k}{2^{s}} \\
& +\sum_{k=-\infty}^{\infty}\left(\frac{\sin z}{z+\pi+2 \pi k}\right)^{2} \prod_{s=0}^{M} \operatorname{tg}^{\alpha_{s+1}} \frac{(z+\pi) / 2+\pi k}{2^{s}} \\
= & \cos ^{2} \frac{z}{2} \operatorname{tg}^{\alpha_{1}} \frac{z}{2} \sum_{k=-\infty}^{\infty}\left(\frac{\sin (z / 2)}{(z / 2)+\pi k}\right)^{2} \prod_{s=1}^{M} \operatorname{tg}^{\alpha_{s+1}} \frac{(z / 2)+\pi k}{2^{s}} \\
& +\sin ^{2} \frac{z}{2} \operatorname{tg}^{\alpha_{1}} \frac{z+\pi}{2} \sum_{k=-\infty}^{\infty}\left(\frac{\sin (z+\pi) / 2}{(z+\pi) / 2+\pi k}\right)^{2} \prod_{s=1}^{M} \operatorname{tg}^{\alpha_{s+1}} \frac{(z+\pi) / 2+\pi k}{2^{s}} .
\end{aligned}
$$

On the assumption of the induction,

$$
\begin{aligned}
\sum_{k=-\infty}^{\infty}\left(\frac{\sin (z / 2)}{(z / 2)+\pi k}\right)^{2} & \prod_{s=1}^{M} \operatorname{tg}^{\alpha_{s}+1} \frac{(z / 2)+\pi k}{2^{s}} \\
& =\sum_{k=-\infty}^{\infty}\left(\frac{\sin (z+\pi) / 2}{(z+\pi) / 2+\pi k}\right)^{2} \prod_{s=1}^{M} \operatorname{tg}^{\alpha_{s+1}} \frac{(z+\pi) / 2+\pi k}{2^{s}} \\
& = \begin{cases}0 & \text { if at least one of } \alpha_{2}, \ldots, \alpha_{M+1} \text { is equal to } 1, \\
1 & \text { otherwise. }\end{cases}
\end{aligned}
$$

Thus

$$
\begin{aligned}
& \sum_{k=-\infty}^{\infty}\left(\frac{\sin z}{z+\pi k}\right)^{2} \prod_{s=1}^{M+1} \operatorname{tg}^{\alpha_{s}} \frac{z+\pi k}{2^{s}} \\
& = \begin{cases}0 & \text { if at least one of } \alpha_{2}, \ldots, \alpha_{M+1} \text { is equal to } 1, \\
\cos ^{2} \frac{z}{2} \operatorname{tg}^{\alpha_{1}} \frac{z}{2}+\sin ^{2} \frac{z}{2} \operatorname{tg}^{\alpha_{1}} \frac{z+\pi}{2} & \text { otherwise. }\end{cases}
\end{aligned}
$$


Since

$$
\cos ^{2} \frac{z}{2} \operatorname{tg}^{\alpha_{1}} \frac{z}{2}+\sin ^{2} \frac{z}{2} \operatorname{tg}^{\alpha_{1}} \frac{z+\pi}{2}= \begin{cases}1 & \text { if } \alpha_{1}=0 \text { or } \alpha_{1}=2, \\ 0 & \text { if } \alpha_{1}=1,\end{cases}
$$

the assertion of the lemma follows from (4.5).

By 'means of Lemma 4.1 it is easy to show that for the validity of identity (4.3) it suffices to find $b_{1}, \ldots, b_{m}$ so that

$$
\sum_{k=-\infty}^{\infty}(g(x+2 \pi k))^{2 n+2}\left(1+\sum_{s=1}^{m} b_{s}^{2} \prod_{p=2}^{s+1} \operatorname{tg}^{2} \frac{x+2 \pi k}{2^{p}}\right) \equiv 1 .
$$

One can easily ascertain that if $0 \leqslant s \leqslant m$ then

$$
\sum_{k=-\infty}^{\infty}(g(x+2 \pi k))^{2 m+2} \prod_{p=2}^{s+1} \operatorname{tg}^{2} \frac{x+2 \pi k}{2^{p}}
$$

is a linear combination of functions $\sin ^{2 s} \frac{x}{2}, \ldots, \sin ^{2 m} \frac{x}{2}$. Hence the finding of $b_{1}^{2}, \ldots, b_{m}^{2}$ from the condition (4.6) brings one to $m \times m$ linear algebraic system (the matrix of this system is triangular); moreover, the specific character of this system is such that $b_{1}^{2}, \ldots, b_{m}^{2}$ satisfying condition (4.6) are uniquely defined and they are positive.

Thus there exists a unique (up to multiplying by -1 ) set of real numbers $b_{1}, \ldots, b_{m}$ such that the function $\psi$ with Fourier image $\psi$ of the form (4.2) satisfies the conditions $\mathrm{A}, \mathrm{B}, \mathrm{C}, \mathrm{D}$ where operators $P$ and $Q$ are of the form (4.1). Prolongations of the type (1.2) generated by the function $\psi$ are piecewise-polynomial functions of the degree at most $m$ for each of the variables.

Remark 4.1. The proved existence of projection-difference analog of operator $B=D_{1}^{2 m}+D_{2}^{2 m}$ coinciding with its simplest $(4 m+1)$-point difference analog may be used for example in the following situation. Let us consider the solution of boundary value problem for equation $L(u)=f$ where $L$ is $2 m$-th order differential operator (generally speaking nonlinear). Under some restrictions on $B$ and $L$ (we assume that functions from the domain of $B$ and $L$ satisfy some boundary conditions [21]) for the solution of projection-difference analog $\hat{L}(\hat{u}) '=\hat{f}$ of the problem $L(u)=f$ effective 2 -stage iterative methods [21] may be used. In spite of the fact that the operator $\hat{L}$ may be of complex structure each step of these methods consists of some iterations in the interior iterative process for the equation $\hat{B} \hat{v}=\hat{F}$ with operator $\hat{B}$ of simple structure (on the use of this approach for 4th order equations see [22], [23]).

\section{$\S 5$. On the interpolation representations of the entire functions of exponential type}

It is necessary and sufficient for the construction of prolongation operators with properties A-D $\S 1$ to construct the function $\psi$ whose Fourier image $\psi$

24 - Banach Center 1. 24 
satisfies the conditions $\mathrm{A}^{\prime}-\mathrm{D}^{\prime}$. In particular $\hat{\psi}$ must be an entire function of exponential type which belongs to $L_{2}\left(E_{n}\right)$ as a function of real $x$ and has zeroes of some multiplicity at the points $2 \pi \alpha, \alpha \in Z_{n}$. Thus it is interesting to receive representations of such functions using values of their derivatives at the points $2 \pi \alpha, \alpha \in Z_{n}$. The well-known Kotelnikov's theorem (see for instance [24]) gives the interpolation representation of entire functions of exponential type $\sigma$ using their values at the points $(\pi \alpha) / \sigma, \alpha \in Z_{n}$. In this section we study the interpolation representations with multiple knotes of the form $2 \pi \alpha$ which are consequences of more general assertions from [25], [26].

For the simplicity of notations we formulate our results only for $n=1$; in the case of arbitrary dimension $n$ these representations (with necessary modification of notations) are also true (see [26] and considerations for $n=2$ at the end of this section). Thus let $n=1$ and let $B_{\sigma}$ be a class of entire functions of exponential type $\sigma$ which belong to $L_{2}\left(E_{1}\right)$ as functions of real $x$. For any natural $N$ and for all integer $s, 0 \leqslant s \leqslant N-1$ let us define polynomials

$$
P_{s N}(x)=\frac{1}{s !} 2^{-s} \frac{d^{s}}{d t^{s}}\left[\left(\frac{t}{\sin t}\right)^{N} e^{2 t x}\right]_{t=0} .
$$

The polynomials $P_{s N}(x)$ are connected with generalized Bernoulli's polynomials ([27], p. 256). There exist representations of $P_{s N}$ which differ from (5.1) (see $[25],[26])$.

LEMma 5.1. Let $f \in B_{\sigma}$ and $N$ be an arbitrary integer satisfying condition $N \geqslant 2 \sigma$. Then

$$
f(z)=\left(2 \sin \frac{z}{2}\right) \sum_{s=0}^{N N-1} \sum_{p \in Z_{1}} \frac{c_{p s}}{(z-2 \pi p)^{N-s}}
$$

where

$$
c_{p s}=(-1)^{p N} P_{s N}(D) f_{0} \cdot(2 \pi p)
$$

$D=\frac{d}{d z}, P_{s N}$ are the polynomials of the form (5.1). Moreover

$$
\sum_{s=0}^{N-1} \sum_{p \in Z_{1}}\left|c_{p s}\right|^{2}<\infty
$$

Conversely if $c_{p s}$ is an arbitrary sequence satisfying the condition (5.4) then (5.2) defines the function $f \in B_{N / 2}$ and the equality (5.3) holds.

The proof of Lemma 5.1 is based on the construction of a special unconditional basis in $L_{2}(-\sigma, \sigma)$; elements of this basis are products of elements of the trigonometric system and derivatives of the Schoenberg $B$-splines (see [25]).

If we use the representation (5.2) for the construction and analysis of PDMs it is necessary to choose such functions $f \in B_{\sigma}$ that have zeroes of some 
multiplicity at the points $2 \pi \alpha$ (see the condition $C^{\prime} \S 1$ ), i.e. we are interested in functions of the form (5.2) such that some of their coefficients $c_{p s}$ are zero (see (5.3)). It is also necessary that the sequence $\left\{c_{p s}\right\}$ should satisfy some restrictions for the condition $B^{\prime}$ to be satisfied. Thus it is possible to describe the subclass of class $B_{\sigma}$ in terms of $c_{p s}$ such that functions of this subclass (and only they) will generate coordinate functions of PDM with the optimal order of approximation. Further analysis of functions of this subclass may go along several lines. For example one of the ways is the construction of PDMs with the optimal rate of convergence on the one hand and their coincidence with simplest difference schemes on the other.

As an example of the application of Lemma 5.1 (more precisely, of its 2-dimensional analog) let us obtain a complete description of entire functions belonging to $B_{1}$ and satisfying the conditions $\mathrm{A}^{\prime}, \mathrm{B}^{\prime}, \mathrm{C}^{\prime} \xi 1$ for $n=2, m=1$. In other words, we shall describe all operators $R$ of the form (1.2) acting from the space of grid functions into $W_{2}^{1}\left(E_{2}\right)$ which are generated by functions $\psi$ with support $\left\{x \in E_{2}:-1 \leqslant x_{k} \leqslant 1, k=1,2\right\}$ and satisfy the approximation property $C \S 1$ in $W_{2}^{1}\left(E_{2}\right)$, i.e. for $m=1, n=2$. This description will be used in Section 6 for a complete description of projection-difference analogs of 2-dimensional Laplace operator.

Let $N=2, \sigma=1$. Then $P_{02}(x)=1, P_{12}(x)=x$ (see (5.1)). Using Lemma 5.1, we find that for any $f \in B_{1}$

$$
\begin{aligned}
f(z)= & \left(2 \sin \frac{z_{1}}{2} \cdot 2 \sin \frac{z_{2}}{2}\right)^{2} \sum_{p \in Z_{2}}\left\{\frac{f(2 \pi p)}{\left(z_{1}-2 \pi p_{1}\right)^{2}\left(z_{2}-2 \pi p_{2}\right)^{2}}\right. \\
& +\frac{D_{1} f(2 \pi p)}{\left(z_{1}-2 \pi p_{1}\right)\left(z_{2}-2 \pi p_{2}\right)^{2}}+\frac{D_{2} f(2 \pi p)}{\left(z_{1}-2 \pi p_{1}\right)^{2}\left(z_{2}-2 \pi p_{2}\right)} \\
& \left.+\frac{D_{1} D_{2} f(2 \pi p)}{\left(z_{1}-2 \pi p_{1}\right)\left(z_{2}-2 \pi p_{2}\right)}\right\} .
\end{aligned}
$$

If we require that

$$
f(2 \pi p)=D_{1} f(2 \pi p)=D_{2} f(2 \pi p)=0
$$

for all $p \in Z_{2}, p \neq(0,0)$ (such is the condition $C^{\prime} \S 1$ for $m=1$ ) then (5.5) implies

$$
\begin{aligned}
\int(z)=\left(4 \sin \frac{z_{1}}{2} \sin \frac{z_{2}}{2}\right)^{2}\left\{\frac{f(0)}{z_{1}^{2} z_{2}^{2}}+\frac{D_{1} f(0)}{z_{1} z_{2}^{2}}\right. & +\frac{D_{2} f(0)}{z_{1}^{2} z_{2}} \\
& \left.+\sum_{p \in Z_{2}} \frac{D_{1} D_{2} f(2 \pi p)}{\left(z_{1}-2 \pi p_{1}\right)\left(z_{2}-2 \pi p_{2}\right)}\right\} .
\end{aligned}
$$

Note that if $f \in B_{1}$ and $\left(1+|x|^{2}\right)^{\frac{1}{2}} \int(x) \in L_{2}\left(E_{2}\right)$ (see the condition $\mathrm{B}^{\prime} \S 1$ for $m=1)$ then $z_{1} f(z) \in B_{1}, z_{2} f(z) \in B_{1}$ because the type of entire function doesn't change after multiplying it by polynomial. By Lemma 5.1, for $z_{1} f(z)$ and $z_{2} f(z)$ and (5.6) we obtain the following representations: 


$$
\begin{aligned}
& z_{1} f(z)=\left(4 \sin \frac{z_{1}}{2} \sin \frac{z_{2}}{2}\right)^{2}\left\{\frac{f(0)}{z_{1} z_{2}^{2}}+\frac{D_{2} f(0)}{z_{1} z_{2}}+\sum_{p \in Z_{2}} \frac{2 \pi p_{1} D_{1} D_{2} f(2 \pi p)}{\left(z_{1}-2 \pi p_{1}\right)\left(z_{2}-2 \pi p_{2}\right)}\right\}, \\
& z_{2} f(z)=\left(4 \sin \frac{z_{1}}{2} \sin \frac{z_{2}}{2}\right)^{2}\left\{\frac{f(0)}{z_{1}^{2} z_{2}}+\frac{D_{1} f(0)}{z_{1} z_{2}}+\sum_{p \in Z_{2}} \frac{2 \pi p_{2} D_{1} D_{2} f(2 \pi p)}{\left(z_{1}-2 \pi p_{1}\right)\left(z_{2}-2 \pi p_{2}\right)}\right\} .
\end{aligned}
$$

Now let us multiply both sides of (5.7) by $z_{1}$ and subtract (5.8) from the obtained equality. Then

$$
0=\left(4 \sin \frac{z_{1}}{2} \sin \frac{z_{2}}{2}\right)^{2}\left\{\frac{D_{1} f(0)}{z_{2}^{2}}+\sum_{p \in Z_{2}} \frac{D_{1} D_{2} f(2 \pi p)}{z_{2}-2 \pi p_{2}}\right\}
$$

which results in $D_{1} f(0)=0$ and

$$
\sum_{p_{1}}^{\infty} D_{1} D_{2} f\left(2 \pi p_{1}, 2 \pi p_{2}\right)=0 \text { for any integer } p_{2} \text {. }
$$

Analogously, from the comparison of (5.7) and (5.9) we have that $D_{2} f(0)=0$ and

$$
\sum_{p_{2}=-\infty}^{\infty} D_{1} D_{2} f\left(2 \pi p_{1}, 2 \pi p_{2}\right)=0 \quad \text { for any integer } p_{1}
$$

Thus (5.7) implies

$$
f(z)=\left(4 \sin \frac{z_{1}}{2} \sin \frac{z_{2}}{2}\right)^{2}\left\{\frac{1}{z_{1}^{2} z_{2}^{2}}+\sum_{p \in Z_{2}} \frac{c_{p}}{\left(z_{1}-2 \pi p_{1}\right)\left(z_{2}-2 \pi p_{2}\right)}\right\}
$$

(here we use the notation $c_{p}=D_{1} D_{2} f(2 \pi p)$ and require that the function $f$ should satisfy the additional condition $f(0)=1$ ) where

$$
\begin{array}{ll}
\sum_{p_{1}=-\infty}^{\infty} c_{p_{1} p_{2}}=0 & \text { for all } p_{2} \in Z_{1}, \\
\sum_{p_{2}=-\infty}^{\infty} c_{p_{1} p_{2}}=0 & \text { for all } p_{1} \in Z_{1} .
\end{array}
$$

Note that

$$
\sum_{p \in Z_{2}}\left(p_{1}^{2}+p_{2}^{2}\right)\left|c_{p}\right|^{2}<\infty
$$

because of (5.8), (5.9) and Lemma 5.1.

It is easy to show that the converse is true, i.e. for any sequence $\left\{c_{p}\right\}$ with the properties $(5.11),(5.12)$ the function $f(z)$ of the form $(5.10)$ satisfies all the necessary conditions.

Thus we have proved the following assertion.

LEMMA 5.2. Let $f(z)$ be an entire function of exponential type 1 satisfying the following conditions: 


$$
\begin{gathered}
\left(1+|x|^{2}\right)^{\frac{1}{2}} f(x) \in L_{2}\left(E_{2}\right), \\
f(0)=1, \quad f(2 \pi p)=D_{1} f(2 \pi p)=D_{2} f(2 \pi p)=0,
\end{gathered}
$$

for all $p \in Z_{2}, p \neq(0,0)$. Then

$$
f(z)=\left(4 \sin \frac{z_{1}}{2} \sin \frac{z_{2}}{2}\right)^{2}\left\{\frac{1}{z_{1}^{2} z_{2}^{2}}+\sum_{p \in Z_{2}} \frac{c_{p}}{\left(z_{1}-2 \pi p_{1}\right)\left(z_{2}-2 \pi p_{2}\right)}\right\},
$$

where

$$
c_{p}=D_{1} D_{2} f(2 \pi p)
$$

Moreover,

$$
\sum_{p \in Z_{2}}\left(p_{1}^{2}+p_{2}^{2}\right)\left|c_{p}\right|^{2}<\infty
$$

and

$$
\begin{array}{ll}
\sum_{p_{1} \in Z_{1}} c_{p_{1} p_{2}}=0 & \text { for all } p_{2} \in Z_{1} \\
\sum_{p_{2} \in Z_{1}} c_{p_{1} p_{2}}=0 & \text { for all } p_{1} \in Z_{1} .
\end{array}
$$

Conversely, if $\left\{c_{p}\right\}$ is an arbitrary sequence satisfying conditions (5.17)-(5.18) then (5.15) defines the function satisfying conditions (5.13), (5.14) and equality (5.16) holds.

\section{§6. Complete description of projection-difference analogs of 2-dimensional Laplace operator}

The purpose of this section is to describe the projection-difference analogs of 2-dimensional Laplace operator generated by the prolongation operator $R$ of the form (1.2) with the function whose support belongs to square $[-1,1] \times[-1,1]$.

More precisely, let $h_{1}=h_{2}=h$ and let $\psi$ be a function satisfying the following conditions (see conditions $A, B, C \S 1$ ):

(1) its support belongs to square $\left\{x \in E_{2}:-1 \leqslant x_{k} \leqslant 1, k=1,2\right\}$;

(2) $\psi \in W_{2}^{1}\left(E_{2}\right)$;

(3) $\psi$ generates the prolongation operator $R$ of the form (1.2) such that for each $u \in W_{2}^{2}\left(E_{2}\right)$ there exists a grid function $\bar{u}$ of the type (1.1) such that

$$
\begin{aligned}
\|\bar{u}\|_{L_{2}\left(E_{2}\right)} & \leqslant K\|u\|_{L_{2}\left(E_{2}\right)}, \\
\|u-R \bar{u}\|_{w_{2}^{s}\left(E_{2}\right)} & \leqslant C_{s} h^{2-s}\|u\|_{W_{2}^{2}\left(E_{2}\right)}, \quad 0 \leqslant s \leqslant 1,
\end{aligned}
$$

where $K$ and $C_{s}$ do not depend on $u$ and $h$.

If $\psi$ is a fixed function satisfying conditions (1)-(3) then it generates operator $Q(\delta)$ such that

$$
(\Delta R \bar{u}, R \bar{v})_{L_{2}\left(E_{2}\right)}=(Q(\hat{\partial}) \bar{u}, \bar{v})_{L_{2}\left(E_{2}\right)}
$$


for all grid functions $\bar{u}, \bar{v}$ (see the condition $\mathrm{D} \S 1$ and Remark 1.3). Note that operator $Q(\hat{\partial})$ defined by $(6.1)$ is projection-difference analog of Laplace operator generated by the function $\psi$ (see Remark 1.4).

It is necessary to find the class of operators $Q(\hat{\partial})$ defined by $(6.1)$ where $\psi$ may move within the set of functions satisfying the conditions (1), (2), (3).

First of all let us note that it follows from Lemma 5.2 that the function $\psi$ satisfies the conditions (1)-(3) if and only if its Fourier image $\hat{\psi}$ is of the following form:

$$
\hat{\psi}(z)=\left(4 \sin \frac{z_{1}}{2} \sin \frac{z_{2}}{2}\right)^{2}\left\{\frac{1}{z_{1}^{2} z_{2}^{2}}+\sum_{p \in Z_{2}} \frac{c_{p}}{\left(z_{1}-2 \pi p_{1}\right)\left(z_{2}-2 \pi p_{2}\right)}\right\} .
$$

Here $\left\{c_{p}\right\}=\left\{c_{p_{1} p_{2}}\right\}$ is an arbitrary sequence such that

$$
\begin{gathered}
\sum_{p_{1} \in Z_{1}} c_{p_{1} p_{2}}=0 \quad \text { for all } p_{2} \in Z_{1}, \\
\sum_{p_{2} \in Z_{1}} c_{p_{1} p_{2}}=0 \quad \text { for all } p_{1} \in Z_{1}, \\
\sum_{p \in Z_{2}}\left(p_{1}^{2}+p_{2}^{2}\right)\left|c_{p}\right|^{2}<\infty ;
\end{gathered}
$$

moreover we assume that the normalization condition $\psi(0)=1$ is satisfied.

Let $\eta \in E_{1}$ and

$$
Q_{\eta}(\hat{\partial})=\hat{\partial}_{1}^{2}+\hat{\partial}_{2}^{2}+\eta h^{2} \hat{\partial}_{1}^{2} \hat{\partial}_{2}^{2}
$$

THEOREM 6.1. For any $\eta \leqslant 1 / 3$ there exists a function $\psi$ with properties (1)-(3) such that operator $Q_{\eta}(\partial)$ of the form (6.6) satisfies condition (6.1). Conversely, for any function $\psi$ with properties (1)-(3) operator $Q(\hat{\partial})$ generated by $\psi$ and equality (6.1) is of the form (6.6) with some $\eta \leqslant 1 / 3$.

Proof. Using Theorem 1.1 and the representation (6.2) we deduce that in order to describe operators $Q(\partial)$ defined by (6.1) it is sufficient to consider the behaviour of the function

$$
\sum_{\alpha \in Z_{2}} F(x+2 \pi \alpha) \quad \text { where } F(x)=-h^{-2}\left(x_{1}^{2}+x_{2}^{2}\right)|\hat{\psi}(x)|^{2} .
$$

It follows from (6.2), (6.3) that

$$
x_{1} \hat{\psi}(x)=\left(4 \sin \frac{x_{1}}{2} \sin \frac{x_{2}}{2}\right)^{2}\left\{\frac{1}{x_{1} x_{2}^{2}}+\sum_{p \in Z_{2}} \frac{2 \pi p_{1} c_{p}}{\left(x_{1}-2 \pi p_{1}\right)\left(x_{2}-2 \pi p_{2}\right)}\right\} .
$$

Then

$$
\begin{aligned}
& \sum_{\alpha_{1} \in Z_{1}}\left(x_{1}+2 \pi \alpha_{1}\right)^{2}\left|\hat{\psi}\left(x_{1}+2 \pi \alpha_{1}, x_{2}\right)\right|^{2} \\
= & \left(4 \sin \frac{x_{1}}{2} \sin \frac{x_{2}}{2}\right)^{4} \sum_{\alpha_{1} \in Z_{1}}\left|\frac{1 / 2}{\left(x_{1} / 2+\pi \alpha_{1}\right) x_{2}^{2}}+\sum_{\substack{p_{1} \in Z_{1} \\
p_{1} \neq 0}} \frac{\pi p_{1}}{x_{1} / 2-\pi p_{1}+\pi \alpha_{1}} \sum_{p_{2} \in Z_{1}} \frac{c_{p}}{x_{2}-2 \pi p_{2}}\right|^{2} .
\end{aligned}
$$


Since

$$
\sum_{k=-\infty}^{\infty} \frac{1}{(y+\pi k)(z+\pi k)}=\frac{\sin (y-z)}{(y-z) \sin y \sin z}
$$

(see, for instance, [20]), we have

$$
\begin{aligned}
\sum_{\alpha_{1} \in Z_{1}}\left(x_{1}+\right. & \left.2 \pi \alpha_{1}\right)^{2}\left|\hat{\psi}\left(x_{1}+2 \pi \alpha_{1}, x_{2}\right)\right|^{2} \\
& =\left(4 \sin \frac{x_{1}}{2} \sin \frac{x_{2}}{2}\right)^{4}\left\{\frac{1 / 4}{x_{2}^{4} \sin ^{2} \frac{x_{1}}{2}}+\left.\left.\sum_{p_{1} \in Z_{1}} \frac{\pi^{2} p_{1}^{2}}{\sin ^{2} \frac{x_{1}}{2}}\right|_{p_{2} \in Z_{1}} \frac{c_{p}}{x_{2}-2 \pi p_{2}}\right|^{2}\right\},
\end{aligned}
$$

whence

$$
\begin{aligned}
& \sum_{\alpha \in Z_{2}}\left(x_{1}+2 \pi \alpha_{1}\right)^{2}|\psi(x+2 \pi \alpha)|^{2} \\
& =4^{3} \sin ^{2} \frac{x_{1}}{2} \sin ^{4} \frac{x_{2}}{2} \sum_{\alpha_{2} \in Z_{1}}\left\{\frac{1 / 16}{\left(\frac{x_{2}}{2}+\pi \alpha_{2}\right)^{4}}+\pi^{2} \sum_{p_{1} \in Z_{1}} p_{1}^{2}\left|\sum_{p_{2} \in Z_{1}} \frac{c_{p}}{\frac{x_{2}}{2}-\pi p_{2}+\pi \alpha_{2}}\right|^{2}\right\} .
\end{aligned}
$$

Using (6.7) and the equality

$$
\sum_{k=-\infty}^{\infty} \frac{1}{(y+\pi k)^{4}}=\frac{1}{\sin ^{4} y}-\frac{2}{3 \sin ^{2} y}
$$

we have

$$
\begin{aligned}
& \sum_{\alpha \in Z_{2}}\left(x_{1}+2 \pi \alpha_{1}\right)^{2}|\hat{\psi}(x+2 \pi \alpha)|^{2} \\
& =4 \sin ^{2} \frac{x_{1}}{2}-\frac{8}{3} \sin ^{2} \frac{x_{1}}{2} \sin ^{2} \frac{x_{2}}{2}+64 \pi^{2} \sin ^{2} \frac{x_{1}}{2} \sin ^{2} \frac{x_{2}}{2} \sum_{p \in Z_{2}} p_{1}^{2}\left|c_{p}\right|^{2} .
\end{aligned}
$$

Analogously

$$
\begin{aligned}
& \sum_{\alpha \in Z_{2}}\left(x_{2}+2 \pi \alpha_{2}\right)^{2}|\hat{\psi}(x+2 \pi \alpha)|^{2} \\
& =4 \sin ^{2} \frac{x_{2}}{2}-\frac{8}{3} \sin ^{2} \frac{x_{1}}{2} \sin ^{2} \frac{x_{2}}{2}+64 \pi^{2} \sin ^{2} \frac{x_{1}}{2} \sin ^{2} \frac{x_{2}}{2} \sum_{p \in Z_{2}} p_{2}^{2}\left|c_{p}\right|^{2} .
\end{aligned}
$$

Thus

(6.8) $\sum_{a \in Z_{2}} F(x+2 \pi \alpha)=-\left(\frac{2}{h} \sin \frac{x_{1}}{2}\right)^{2}-\left(\frac{2}{h} \sin \frac{x_{2}}{2}\right)^{2}$

$$
+\frac{16}{h^{2}} \sin ^{2} \frac{x_{1}}{2} \sin ^{2} \frac{x_{2}}{2}\left\{\frac{1}{3}-4 \pi^{2} \sum_{p \in Z_{2}}\left(p_{1}^{2}+p_{2}^{2}\right)\left|c_{p}\right|^{2}\right\} \text {. }
$$


Let us calculate now the function $G(x)$ corresponding to difference operator $Q_{\eta}(\hat{\partial})$ of the form (6.6) (see (1.9) and Remark 1.6):

$$
G(x)=-\left(\frac{2}{h} \sin \frac{x_{1}}{2}\right)^{2}-\left(\frac{2}{h} \sin \frac{x_{2}}{2}\right)^{2}+\eta \cdot \frac{16}{h^{2}} \sin ^{2} \frac{x_{1}}{2} \sin ^{2} \frac{x_{2}}{2}
$$

From the comparison of (6.8) and (6.9) we deduce that

$$
\sum_{\alpha \in Z_{2}} F(x+2 \pi \alpha)=G(x)
$$

if and only if

$$
\eta=\frac{1}{3}-4 \pi^{2} \sum_{p \in Z_{2}}\left(p_{1}^{2}+p_{2}^{2}\right)\left|c_{p}\right|^{2} .
$$

It follows from (6.10) that by choosing $\left\{c_{p}\right\}$ with properties (6.3)-(6.5) we may obtain any $\eta$ from $\left(-\infty, \frac{1}{3}\right]$.

Thus, on the one hand, every projection-difference analog of Laplace operator is of the form (6.6) with $\eta \leqslant \frac{1}{3}$ and, on the other hand, every difference operator of the form (6.6) with $\eta \leqslant \frac{1}{3}$ may be constructed by PDM with the function $\psi$ satisfying conditions (1)-(3). Theorem 6.1 is proved.

Remark 6.1. The theorem asserts that none of the operators of the form (6.6) with $\eta>\frac{1}{3}$ is generated by PDM with coordinate functions satisfying the conditions (1)-(3). Apparently there exists some interior property of operators of the form (6.6) differentiating classes $\eta \leqslant \frac{1}{3}$ and $\eta>\frac{1}{3}$.

Remark 6.2. By weakening the restrictions concerning $\psi$ we may construct operators of the form (6.6) with $\eta \in\left(\frac{1}{3}, \frac{1}{2}\right)$ by means of PDM. It may be achieved at the expense of both optimal approximation in weaker norms and extension of the support of function $\psi$ (see [12]).

\section{References}

[1] G. Strang and G. J. Fix, An Analysis of the Finite Element Method, Prentice-Hall, Inc. 1973.

[2] А. Н. Тихонов and А. А. Самарский, Об однородиых разиостиых схемах, ЖВМ и МФ । (1) (1961), 5-63.

[3] С. Г. Михлин, Вариачиопиве Memodы в Mameмanической Физике, Наука, Москва 1970.

[4] R. Courant, Variational methods for the solution of problems of equilibrium and vibrations, Bull. Amer. Math. Soc. 49 (1) (1943), 1-23.

[5] F. di Guglielmo, Construction d'approximations des espaces de Sobolev sur des réseaux en simplexes, Calcolo, 6 (2) (1969), 279331.

[6] Н. А. Стрелков, Сииплициальные распространсния сеточиых буикций и их применение для решения задич математической физики, ЖВМ и МФ II (4) (1971), 969.981.

[7] -.О6 аппроксимачиях иекоторых функционалылых прострапств и их применени.. ДАН СССР 209 (3) (1973), 565-568. 
[8] -, Об аппроксимачиях некоторых функциональных пространств и их применениях 8 теории проекционио-разностных схем, Дисс. канд. физ.-мат. наук, Москва, МГУ 1973.

[9] -, Об аппроксимачии пространств типа С. Л. Соболева, Вестннк Ярославского ун-та 10 (1974), 123-156.

[10] -, Об изометрических восполиенилх сеточных функиий, Труды I конференции молодых ученых ф-та вычисл. матем. и кибернетики МГУ, Москва, МГУ 1973, 55-61.

[11] -, Изометричиость операторов восполиения сетqиных функций, вариачионно-разно. стные методы в математической физике, Новосибирск, ВЦ СО АН СССР 1974, 74-83.

[12] -, О проекционно-разностиых аналогах операпора Лапласа, ЖВМ и МФ 21 (5) (1981), $1326-1328$.

[13] -, О совокуниости операторов о разиостных и проекиионио-разностиых методах, Bариачиони-раностные методы в мателяатической физике, Новосибирск, ВЦ СО АН CCCP 1976, 73-78.

[14] -, О выборе координатных функций в проскционно-разностиых методах, ЖВМ и МФ 17 (6) (1977), 1443-1457.

[15] J.-P. A ubin, Approximation of Elliptic Boundary-Value Problems, Wiley, New York 1972.

[16] С. М. Нихольский, Приближение Функиий Многих Переменных и Теоремы Вложения, Наука, Москва 1977.

[17] J.-L. Ljons and E. Magenes, Problèmes aux Limites non Homogènes et Applications, Dunod, Paris 1968.

[18] G. Strang and G. Fix, A Fourier analysis of the finite element method, Proc. CIME Summer School, Italy 1971.

[19] R. S. Varga, Functional analysis and approximation theory in numerical analysis, SIAM, Philadelphia 1971.

[20] И. С. Градштейн и И. М. Рыжик, Таблицы Иитегралов, Сумм, Рядов и Произведений, Наука, Москва 1971.

[21] Е: Г. Дьяконов, Разностиые Методы Решения Краевых Задач, вып. 1, Москва, МГУ 1971.

[22] Н. А. Стрелков, Итерачионные методы решения проекиионно-разиостных аналогов краевых задач для линейных и квазилиейпых уравнений четөертого порядка, ЖВМ и МФ (19 (1) (1979), 143-155.

[23] -, Имерачионные методы речения проекционо-разиостиых аналогов краевых задач для уравиений четверпого порядка, Вариачионо-разюостные методы в математической физике, Новосибирск, ВЦ СО АН СССР 1978, 165-177.

[24] Б. Я. Л вин, Целые- функции (курс лекций), Москва, Ин-т механики МГУ 1971.

[25] Н. А. Стрелков, Сплайн-тригонометрические базисы о $\mathrm{L}_{2}$ и интерполяция целых бункций экспоненциалыного типа, Мат. Заметки 32 (6) (1982), 835-840.

[26] -, Нитерполячониые представления цельх фуикцйи экспоненииального типа, Методы аппраксимачии и иттерполячии, Новосибирск, ВЦ СО АН СССР 1981, 144-147.

[27] Г. Бейтмен и А. Эрдейи, Высиие Трансцендентные Функци. Элыпитические и Авмоморфиые Функции. Фуикини Ламе и Матье, Наука, Москва 1967. 